\title{
MS13-03 | New Complexes Of Silver(I) With Azoles
}

Checinska, Lilianna (University of Lodz, Faculty of Chemistry, Lodz, POL); Komerist, Yevgeniy (University of Lodz, Faculty of Chemistry, Lodz, POL); Ochocki, Justyn (Medical University of Lodz, Department of Bioinorganic Chemistry, Lodz, POL)

This research project is a part of our ongoing studies concerning the biological activity of new silver(I) compounds, which have drawn much attention as potential antimicrobial agents.

We determined crystal and molecular structures of silver(I) complexes with azole ligands (as clotrimazole, fluconazole etc). Azole agents are widely used in medicine due to their antifungal and antibacterial activity. Complexes of azoles with biometals (eg. with $\mathrm{Ag}$ ) may extend the range of potential drugs and affect the diversity of their properties, selectivity and efficiency.

The next step is to determine whether the obtained complexes are effective antimicrobial agents and whether they represent a possible alternative to commonly used drags. 